${ }^{1}$ Centro de Tecnologias para o SUS - São Paulo (SP), Brasil. fotini.toscas@isaude. sp.gov.br

2 Santa Casa de Porto Alegre - Porto Alegre (RS), Brasil.

${ }^{3}$ Associação Brasileira de Engenharia Clínica (ABEClin) - Manaus (AM) Brasil.

\section{Mulheres na gestão de tecnologias e engenharia clínica: o caso dos ventiladores pulmonares na Covid-19}

\author{
Women in technology management and clinical engineering: the case \\ of pulmonary ventilators at Covid-19
}

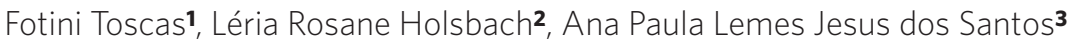

DOI: 10.1590/0103-11042021E117

\begin{abstract}
RESUMO O risco iminente de desabastecimento de ventiladores pulmonares nos serviços de saúde acarretou diversas frentes de trabalho para disponibilizar o maior número possível desses equipamentos para o tratamento dos pacientes acometidos. O agravamento da crise sanitária colapsou serviços de saúde com busca isocrônica por leitos. Em meio ao colapso, foi detectada nova variante da linhagem Sars-CoV-2 e confirmado o primeiro caso de reinfecção. Entre os pontos críticos, foi destaque a escassez caótica de oxigênio e taxas de ocupação de leitos acima de $90 \%$. Pretende-se relatar a participação das autoras nas iniciativas para o enfrentamento da pandemia de relevância internacional. Destacar a participação de mulheres em atividades que são cruciais para responder, em tempo oportuno, às demandas oriundas de emergências sanitárias. Por meio de método de pesquisa de abordagem descritiva e exploratória, buscou-se verificar o perfil das mulheres atuantes na gestão de tecnologias no enfrentamento da pandemia. Destaca-se a pesquisa recente da Associação Brasileira de Engenharia Clínica que verificou que apenas $19 \%$ dos associados respondentes eram mulheres, enquanto $81 \%$ eram do sexo masculino. Assim, divulgar e dar amplo conhecimento das ações de mulheres nessa área pode colaborar para o alcance da igualdade de gênero e empoderar todas as mulheres e meninas.
\end{abstract}

PALAVRAS-CHAVE Aprovação de equipamentos. Acesso a medicamentos essenciais e tecnologias em saúde. Pandemias. Gestão em saúde.

ABSTRACT The imminent risk of shortage of pulmonary ventilators in health services has resulted in several work fronts to maintain and make available the largest possible number of equipment available for the treatment of patients. The worsening of the health crisis has collapsed health services with an isochronic search for beds. Amid the collapse, a new variant of the Sars-CoV-2 strain was detected and the first case of reinfection was confirmed. Among the critical points was the chaotic oxygen scarcity and bed occupancy rates above $90 \%$. We intend to report the participation of the authors in the initiatives to face the pandemic; highlight the participation of women in activities that are crucial to respond, in a timely manner, to the demands arising from health emergencies. Through a research method with a descriptive and exploratory approach, we sought to verify the profile of women working in the management of technologies in facing the pandemic. The recent survey by the Brazilian Association of Clinical Engineering stands out, which found that only $19 \%$ of the respondent associates were women. Thus, disseminating and giving broad knowledge of women's actions in this area can collaborate in achieving gender equality and empower all women and girls.

KEYWORDS Device approval. Access to essential medicines and health technologies. Pandemics. Health management. 


\section{Introdução}

A crise sanitária imposta pela pandemia da Covid-19 tem exigido esforços hercúleos dos sistemas de saúde. Nesse enfrentamento, veio à tona das discussões a oferta e a disponibilidade de ventiladores pulmonares, equipamento médico-assistencial crucial para o atendimento dos casos graves da doença. A assimetria entre a disponibilidade desses equipamentos ante a demanda histórica mundial gerou disputas entres os países, com restrições e vedações de exportação, como a Lei $n^{0}$ 13.993/20201, que proibiu a exportações de ventiladores pulmonares e outros produtos essenciais ao combate à epidemia de coronavírus no Brasil.

$O$ ventilador pulmonar é um equipamento utilizado para proporcionar a ventilação pulmonar e artificial. O objetivo é prover suporte ventilatório completo ou parcial em pacientes que não conseguem respirar por vias normais.

$\mathrm{O}$ risco iminente de desabastecimento de ventiladores pulmonares nos serviços de saúde acarretou diversas frentes de trabalho e forças tarefas para manter e disponibilizar o maior número possível desses equipamentos à disposição para o tratamento dos pacientes acometidos pela doença. Os esforços foram empregados na escalada da produção nacional, em iniciativas para realizar manutenção e recuperar o maior número possível de ventiladores pulmonares, e iniciativas de projetos de pesquisa, desenvolvimento e inovação de novos equipamentos. Tendo em vista a complexidade tecnológica e operacional desses equipamentos, surgiu a necessidade de destacar a importância da atuação dos profissionais de gestão de tecnologias em saúde e da engenharia clínica.

$\mathrm{O}$ agravamento da crise sanitária, em janeiro de 2021, colapsou serviços de saúde com busca isocrônica por Unidades de Terapias Intensivas (UTI). Em meio ao colapso, a Fundação Oswaldo Cruz (Fiocruz) detectou nova variante da linhagem Sars-CoV-2 e confirmou o primeiro caso de reinfecção pelo novo coronavírus no estado do Amazonas. Entre os pontos críticos, foram destaque: a escassez caótica de oxigênio e as taxas de ocupação de leitos de UTI acima de $90 \%{ }^{2}$.

A extrema urgência no abastecimento das unidades de saúde provocou ações conjuntas entre a sociedade civil e o poder público. Entre elas, destacou-se, inclusive em âmbito nacional, a 'SOS AM'. A ação proveu retorno ágil à população (individual, com fornecimento e abastecimento de cilindros) e prioritariamente à rede de saúde ${ }^{3}$. Um diferencial na ação foi a agilidade dos processos e a mobilização já no início de profissionais de engenharia clínica. Esses profissionais, incluindo autoras deste estudo, participaram de todo o processo, desde a aquisição até a instalação de equipamentos médicos, principalmente ventilador pulmonar, além da atuação na análise contingencial para suprir o oxigênio, prestando suporte técnico na aquisição e gestão tecnológica de concentradores e usinas de oxigênio.

A gestão de tecnologias em saúde é definida na Política Nacional de Gestão de Tecnologias em Saúde (PNGTS), publicada em 2011 pelo Ministério da Saúde ${ }^{4}$, como um conjunto de atividades gestoras relacionadas com os processos de avaliação, incorporação, difusão, gerenciamento da utilização e retirada de tecnologias do sistema de saúde. Esse processo deve ter como referenciais as necessidades de saúde, o orçamento público, as responsabilidades dos três níveis de governo e do controle social, além dos princípios de equidade, universalidade e integralidade, que fundamentam a atenção à saúde no Brasil.

Já a engenharia clínica pode ser definida como o ramo da engenharia dedicado a auxiliar e mesmo interferir na área da saúde, com o intuito de alcançar bem-estar, segurança, redução de custos e qualidade nos serviços disponíveis aos pacientes e à equipe multidisciplinar do hospital, por meio da aplicação dos conhecimentos gerenciais e de engenharia à tecnologia da área de saúde. Esses profissionais agem em todo o ciclo de vida das tecnologias da saúde, desde a Pesquisa, Desenvolvimento e Inovação (PD\&I), gerenciamento da tecnologia 
médico-hospitalar, avaliação de tecnologias em saúde, incorporação e aquisição de tecnologias, gestão regulatória e acesso de mercado.

A atuação das mulheres na área de gestão de tecnologias e engenharia cresce paulatinamente. No que tange à engenharia, principalmente nas atuações em campo e cargos de alta gestão, ainda é correlacionada com atuações masculinas; o que torna a abordagem do tema de maior relevância ao destaque das mulheres na área de engenharia clínica ${ }^{5}$.

Para discutir o tema, realizou-se uma pesquisa, por meio de formulário eletrônico detalhado na seção de Métodos, para conhecer e compreender a participação das mulheres nas áreas de ciência e saúde, com foco em engenharia clínica. Foram respondidos 102 formulários, em que após refinamento, foram validados 88 destes. Após novo refinamento, obtiveram-se 81 formulários de mulheres com curso superior que estavam atuando com o uso de tecnologias para a saúde no apoio a pandemia de Covid19. Destas, apenas 56 realizaram o Curso de Especialização em Engenharia Clínica.

Em 2020, a Associação Brasileira de Engenharia Clínica (ABEClin) realizou pesquisa com o objetivo de conhecer o salário de seus associados (todos que trabalham com engenharia clínica). Um dos questionamentos foi o sexo, e apenas 19\% dos associados que responderam o formulário eram mulheres, enquanto $81 \%$ eram do sexo masculino, de um universo de 261 formulários respondidos. Isso permite dizer que o número de mulheres na engenharia clínica, ou seja, que atuam com tecnologias da saúde é inferior de forma significativa ao sexo masculino ${ }^{6}$.

$\mathrm{Na}$ atuação direta para o enfrentamento da pandemia, a ABEClin, em parceria com o Ministério da Saúde, o Ministério da Economia e o Serviço Nacional de Aprendizagem Industrial (Senai), realizou um levantamento de voluntários para atuar na força tarefa para manutenção de ventiladores pulmonares ${ }^{\mathbf{7}, \mathbf{8}}$, em que, do total de 118 voluntários profissionais, somente $20 \%$ eram mulheres.

Pretende-se relatar a participação das autoras no campo de gestão de tecnologias essenciais para o enfrentamento da pandemia de relevância internacional decorrente do novo coronavírus humano. Destacar a participação de mulheres em atividades que são cruciais para responder, em tempo oportuno, às demandas oriundas de emergências sanitárias. Registrar e evidenciar a atuação de mulheres na gestão tecnológica com vistas ao alcance, estímulo e recrutamento de maior participação de mulheres nas atividades de engenharia clínica e gestão de tecnologias em saúde.

\section{Material e métodos}

O método de pesquisa escolhido para o levantamento dos dados foi descritivo e exploratório para atender a uma questão específica por meio de buscas na literatura. Nesse caso, a questão à qual se pretende responder corresponde à Covid-19. A busca na literatura foi feita na base de dados disponíveis no sítio eletrônico do Ministério da Saúde, com os dados da resposta brasileira ao enfrentamento do novo coronavírus, e da página eletrônica da ABEClin, com as atividades de suporte à Covid19. Foram verificadas as ações registradas, além de pesquisadas cinco perguntas fechadas entre abril e julho de 2020 utilizando os descritores combinados pelo operador lógico ‘Covid-19' e 'ventilador pulmonar'. O símbolo de asterisco foi empregado para incluir na pesquisa o termo no plural. Os termos foram buscados no campo que inclui na pesquisa os trabalhos que possuem o termo no título, resumo ou palavras-chaves, possibilitando a localização de um maior número de documentos. Foi encontrado um total de cinco documentos. O quadro 1 mostra a distribuição dos artigos encontrados, e o quadro 2, as referências das buscas realizadas.

A pesquisa foi limitada para apenas artigos científicos, em que foi obtido um total de 13 documentos. Após, foi realizada a leitura do título, do resumo e das palavras-chave de todos os trabalhos, em que foram 
selecionados cinco artigos, classificando-os conforme o objeto de estudo.

A pesquisa pode ser classificada como de natureza aplicada, pois tem por objetivo gerar conhecimentos para aplicação prática. Quanto à abordagem, a pesquisa é tanto qualitativa quanto quantitativa, pois envolve um aprofundamento da compreensão de uma organização ao mesmo tempo que recorre à linguagem matemática para descrever as causas de um fenômeno. A utilização conjunta das pesquisas qualitativa e quantitativa permite obter mais informações do que se fossem feitas de forma isolada, corroborando as experiências práticas das autoras nos processos de gestão de tecnologias. O objetivo é proporcionar maior familiaridade com o problema com vistas a torná-lo explícito ou a construir hipótese, e de procedimento bibliográfico, elaborada com base em material já publicado, ${ }^{9,10}$.

O relato (quadro 3) apresenta as recentes experiências das autoras em iniciativas para o enfrentamento da pandemia da Covid-19, na gestão tecnológica do ventilador pulmonar considerado equipamento essencial para o combate da pandemia.

Quadro 1. Lista dos artigos utilizados no estudo

\begin{tabular}{ll}
\hline Título & Referência \\
\hline $\begin{array}{l}\text { Dispõe sobre a proibição de exportaç̃̃es de produtos médicos, hospitalares e de } \\
\text { higiene essenciais ao combate à epidemia de coronavírus no Brasil. }\end{array}$ & $\begin{array}{l}\text { Brasil. Lei no 13.993, de 23 de } \\
\text { abril de 20201 }\end{array}$ \\
$\begin{array}{l}\text { Política Nacional de Gestão de Tecnologias em Saúde (PNGTS) } \\
\text { Mulheres em carreiras de prestígio: conquistas e desafios à feminização. Cader- } \\
\text { nos de Pesquisa v. } 47 \text { n. } 163 \text { p. 10-14 jan./mar. } 2017\end{array}$ & $\begin{array}{l}\text { Brasil, 20114 } \\
\text { Lombardi MR, 20175 }\end{array}$ \\
$\begin{array}{l}\text { Assessing the impact of coordinated COVID-19 exit strategies across Europe } \\
\text { Deep immune profiling of COVID-19 patients reveals distinct immunotypes with } \\
\text { therapeutic implications. }\end{array}$ & N. WRuktanonchai et al. ${ }^{\mathbf{1 1} .}$ \\
\hline
\end{tabular}

Fonte: Elaboração própria.

Quadro 2. Levantamentos de dados

\begin{tabular}{ll}
\hline Levantamento de Dados & Referência \\
\hline Ministério da Saúde - Brasil & https://coronavirus.saude.gov.br/resposta-brasileira-a-emergencia \\
Associação Brasileira de Engenharia & http://www.abeclin.org.br/post.php?p=213 \\
Clínica (ABEClin) & \\
\hline
\end{tabular}

Fonte: Elaboração própria.

Quadro 3. Relatos de experiência na pandemia

\begin{tabular}{ll}
\hline $\begin{array}{l}\text { Relatos de experiências na Pandemia com o suporte técnico das engenheiras } \\
\text { clínicas }\end{array}$ & Observação \\
\hline R1 - Relato 1- A engenharia clínica proveu suporte técnico para as montadoras & As montadoras automobilísticas \\
$\begin{array}{ll}\text { automobilísticas montarem os laboratórios para consertar os ventiladores pul- } \\
\text { monares e consultoria técnica voluntária para os processos de consertos e reparos }\end{array}$ & e a Rede Senai estão realizando o \\
trabalho de forma voluntária \\
R2 - Relato 2 - Participação de engenheiros clínicos (pessoa física e jurídica) no & Mídias digitais da ABEClin (Insta- \\
conserto de ventiladores pulmonares. Captação de voluntários com o conheci- & $\begin{array}{l}\text { gram/Facebook/Linkedln/Grupo } \\
\text { mento técnico sobre ventiladores. }\end{array}$ \\
& Ministério da Saúde - Brasil \\
& Ministério da Economia - Brasil
\end{tabular}


Quadro 3. (cont.)

\begin{tabular}{ll}
\hline $\begin{array}{l}\text { Relatos de experiências na Pandemia com o suporte técnico das engenheiras } \\
\text { clínicas }\end{array}$ & Observação \\
\hline R3 - Relato 3 - Participação da Gestão de Tecnologias para mapear o mercado & Ministério da Saúde - Brasil \\
e estudo setorial de ventiladores pulmonares para subsidiar ações de escalada & Ministério da Economia - Brasil \\
produtiva & \\
R4 - Relato 4 - Participação da Gestão de Tecnologias no acompanhamento de & Ministério da Saúde - Brasil \\
projetos de pesquisa, desenvolvimento e inovação de novos ventiladores pulmo- & Ministério da Ciência, Tecnologia \\
nares & e Inovação - Brasil \\
& Ministério da Economia - Brasil \\
& Agência Brasileira de Desenvolvi- \\
& mento Industrial - ABDI \\
& Agência Nacional de Vigilância \\
& Sanitária - Anvisa \\
\hline
\end{tabular}

Fonte: Elaboração própria.

De maneira complementar, foi realizada pesquisa entre 15 de julho 2020 e 15 de agosto 2020, aplicada por meio da plataforma Google Forms, com o propósito de conhecer e compreender a atuação das mulheres nas áreas de ciência e saúde, com foco em engenharia clínica e a atuação na pandemia de Covid19. O formulário foi elaborado por meio da ferramenta on-line no Google Forms, com perguntas semiabertas. O link foi encaminhado às profissionais da área de engenharia clínica e divulgado nas mídias digitais (Instagram, Facebook, LinkedIn, WhatsApp e Telegram) da ABEClin, grupos de engenharia clínica no Facebook e WhatsApp, nos links https://docs. google.com/forms/d/e/1FAIpQLSdpvD8fy8M Z0jyP0Uc6rOz5CshvdkvDAgt7x9KRRjYCW VJ03A/viewform?usp=sf_link e https://forms. gle/CzRVWEelgtYepcrM8.

A pesquisa foi limitada a 102 formulários. Após, foi realizado um refinamento em que foram selecionados 88 formulários, classificando-os conforme o objeto de estudo. Os demais formulários foram excluídos da pesquisa por não tratarem de informações consistentes.

\section{Resultados e discussão}

Os relatos relacionados referem-se às atividades realizadas na esfera do governo federal, nos serviços de saúde e em associação de classe de engenharia clínica.

$\mathrm{Na}$ esfera federal, para subsidiar e contribuir para a escala produtiva da indústria nacional de ventiladores pulmonares, foi realizado estudo inicial do mercado com o mapeamento dos registros sanitários válidos de fornecedores nacionais e internacionais com configurações técnicas requeridas para o tratamento da Covid-19, contendo o levantamento dos dados com os principais gargalos no processo de produção nacional e a identificação dos componentes críticos, além das informações sobre a capacidade produtiva. A escala produtiva exigiu esforços governamentais, arranjos empresariais e industriais, arranjos financeiros e estudos de situações patentárias. O objetivo da escalada é promover o aumento exponencial da produção mensal das indústrias nacionais, com registros sanitários válidos de ventiladores pulmonares, para atender à demanda assistencial em crescimento exponencial.

Além das iniciativas envidadas para a escala produtiva dos ventiladores pulmonares já consagrados no mercado nacional, a atuação na gestão de tecnologias amparou as ações governamentais para apoiar projetos de PD\&I de novos ventiladores pulmonares. Destaca-se a importância desse processo, já que o projeto de desenvolvimento requer especificações técnicas e dados de entradas específicos, além 
da gestão de riscos com as análises dos componentes, em atenção à gravidade pulmonar dos pacientes em tratamento da Covid-19. Os projetos devem obrigatoriamente observar os aspectos de regularidade sanitários, porém, dada a complexidade do equipamento, outros aspectos devem ser analisados para disponibilização nos serviços de saúde, como características do gerenciamento da tecnologia durante todo o ciclo de vida, como treinamento, manutenção, assistência técnica, peças de reposição, fornecimento dos consumíveis, desativação e descarte. Ressalta-se que o ventilador pulmonar é tecnologia operador-dependente, cujas características de usabilidade, treinamento, interface são consideradas para minimizar a curva de aprendizado. A fim de otimizar os recursos públicos, são observadas as versatilidades das configurações para que os ventiladores pulmonares possam ser aproveitados nos serviços de saúde em outras condições clínicas no período pós-pandemia. Assim, foram realizadas análises dos projetos com prontidão tecnológica e passíveis de escalonamento tendo como desafio transformar os protótipos viáveis e funcionais, com as orientações de aspectos regulatórios, e técnico-operacionais, com os critérios mínimos que assegurem a qualidade e segurança dos ventiladores pulmonares.

A atuação da gestão de tecnologias também colaborou nos processos para avaliação comparativa das especificações técnicas dos ventiladores pulmonares, por meio de referências técnicas como o documento 'Technica lspecifications for invasiveand non-invasive ventilators for Covid-19 Interim gui-

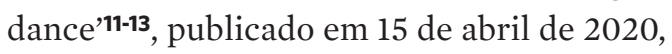
pela Organização Mundial da Saúde (OMS). A avaliação se estendeu aos projetos de desenvolvimento de ventiladores pulmonares com prontidão tecnológica com o objetivo de apoiar avaliações precoces de novas tecnologias, além de contribuir tecnicamente nas análises referentes às medidas tributárias para alterações tarifárias, restrições às exportações dos equipamentos críticos para enfrentamento da pandemia.
Outro considerável fato foi a análise técnica de ventiladores desenvolvidos em parceria entre indústria e poder público. A indústria buscou relação custo $\mathrm{x}$ benefício para produção em escala, o que demonstrou o notório diferencial na avaliação por um especialista na área de engenharia clínica. Nesse exemplo, a análise confirmou que o equipamento desenvolvido não teria mercado para consumo em médio e longo prazo.

Outra participação em destaque é na força-tarefa para manutenção dos ventiladores pulmonares, que reúne mais de 500 engenheiros e técnicos capacitados voluntários, mais de 21 parceiros, e está distribuída por toda a federação pronta para prestar o suporte necessário para recolocar em operação o maior número de ventiladores pulmonares. Estima-se que estão fora de operação 3,6 mil ventiladores pulmonares que impactam nos serviços de saúde, sendo mais de 2 mil equipamentos recuperados por essa iniciativa 7,8 .

Na ação da força-tarefa, a ABEClin participou com duas relevantes linhas de frente: na captação de voluntários profissionais (pessoa física e jurídica) e na orientação às entidades responsáveis na preparação do local para a realização da manutenção dos ventiladores pulmonares. A orientação ocorreu em toda a cadeia de processo, desde o recolhimento dos equipamentos nos Estabelecimentos Assistenciais de Saúde (EAS), recebimento no local da manutenção, higienização, manutenção, calibração até o retorno. Já foram devolvidos mais de 1.500 ventiladores pulmonares.

A captação de voluntários ocorreu totalmente on-line por meio das mídias digitais da ABEClin (Instagram/Facebook/LinkedIn/ Grupo oficial WhatsApp e do Telegram). A relação dos voluntários foi encaminhada ao Ministério da Saúde e ao Ministério da Economia para operacionalização da força-tarefa ${ }^{8}$.

Os comunicados de orientação para as empresas terceirizadas para serviços de engenharia clínica e profissionais no manejo do ventilador pulmonar, a busca de troca de 
experiência internacional com os demais colegas da área e a atuação constante no combate a desinformação também foram destaque.

Entre tantas trocas de experiências internacionais, ressalta-se a entrevista realizada com a Associação Italiana de Engenharia Clínica (Associazione Italiana Ingegneri Clinici AIIC) em março de 2020. A realização das manutenções preventivas e corretivas dos equipamentos médicos, principalmente utilizados na UTI foi uma das principais orientações de nossos colegas italianos. Outro ponto de relevância na entrevista foi a orientação para a engenharia clínica brasileira aproveitar a oportunidade de informação antecipada em um momento com dados escassos para a tomada de decisão, sendo um diferencial para a preparação dos profissionais no enfrentamento da Covid-19.

O estado emocional foi uma das preocupações; parcerias com entidades por meio de projetos como a escuta ativa colaboraram para proporcionar alívio aos profissionais nos serviços de saúde.

$\mathrm{Na}$ análise e no tratamento dos dados da pesquisa realizada com os 88 formulários válidos, foi possível verificar que 81 mulheres possuíam curso superior e estavam atuando na gestão de tecnologias para a saúde no apoio à pandemia de Covid-19. Quanto à formação acadêmica, observou-se que 56 responderam que tinham o Curso de Especialização em Engenharia Clínica, 2 com formação em cursos técnicos de nível médio e 5 não informaram. Em relação ao tempo de formação, menos de 1 ano foi 17,11\%; já entre 1 ano e 5 anos de formada, foi obtido $14,47 \%$; e acima de 5 anos, 28,95\%. Em relação ao tempo de atuação até 1 ano, 15,91\%; entre 1 ano e 5 anos, $28,41 \%$; e acima de 5 anos, $46,59 \%$. Os locais de atuação mais significativos foram em serviços de saúde, 56,03\%; empresas privadas, $21,28 \%$; área pública, 9,22\%; e área acadêmica, 5,67\%.

\section{Considerações finais}

A crise causada pela pandemia do novo coronavírus humano despontou as discussões para a necessidade de profissionais que atuem na gestão de equipamentos médico-hospitalares em todas as esferas de gestão - e, especialmente, nos serviços de saúde. Esses profissionais, operantes em gestão de tecnologias e engenharia clínica, ainda são, em sua maioria esmagadora, homens. A participação das mulheres nesses cenários é altamente relevante, muito embora ainda tímida pelo pouco espaço ocupado. Divulgar e dar amplo conhecimento as ações de mulheres nessa área, criar ambientes favoráveis e estimular para que os espaços das engenharias de saúde e cargos de gestão possam ser ocupados por mulheres em posição de igualdade colaboram para o cumprimento do Objetivo 5 da Agenda 2030 para o Desenvolvimento Sustentável: alcançar a igualdade de gênero e empoderar todas as mulheres e meninas.

\section{Colaboradoras}

Toscas F (0000-0002-6447-2045)* contribuiu para a concepção e planejamento; elaboração do rascunho e da versão final do manuscrito. Holsbach LR (0000-0002-9591-132X)* contribuiu para a metodologia, análise e interpretação dos dados e revisão. Santos APLJ (0000-0003-2598-1549)* contribuiu para a análise e interpretação dos dados. 


\section{Referências}

1. Brasil. Lei ${ }^{\circ}{ }^{13.993}$, de 23 de abril de 2020 , que dispõe sobre a proibição de exportações de produtos médicos, hospitalares e de higiene essenciais ao combate à epidemia de coronavírus no Brasil. Diário Oficial da União. 24 Abr 2020.

2. Lobato F. Amazonas: Fiocruz detecta nova linhagem da Sars-CoV-2 e estado sofre com falta de oxigênio. [acesso em 2021 maio 19]. Disponível em: https://portal.fiocruz.br/noticia/amazonas-fiocruz-detecta-nova-linhagem-da-sars-cov-2-e-estado-sofre-com-falta-de-oxigenio.

3. Mansueto LF. Cecon inaugura usina de oxigênio doada pelo SOS AM. [acesso em 2021 maio 19]. Disponível em: http://www.saude.am.gov.br/visualizar-noticia.php?id=6009.

4. Brasil. Ministério da Saúde. Política Nacional de Gestão de Tecnologias em Saúde. 1. ed., 1. reimpr. Brasília, DF: MS; 2011. 48 p.

5. Lombardi MR. Mulheres em carreiras de prestígio: conquistas e desafios à feminização. Cad. Pesq. 2017; 47(163):10-14.

6. Associação Brasileira de Engenharia Clínica. Pesquisa Salarial 2020. [acesso em 2021 maio 19]. Disponível em: http://www.abeclin.org.br/post.php?p=160.

7. Portal da Indústria. Senai e indústrias fazem manutenção de respiradores mecânicos. [acesso em 2020 jun 15]. Disponível em: http://www.portaldaindus- tria.com.br/canais/industria-contra-covid-19/iniciativas/senai-e-industrias-fazem-manutencao-de-respiradores-mecanicos/.

8. Associação Brasileira de Engenharia Clínica. Links e documentos covid-19. [acesso em 2020 jun 15]. Disponível em: http://www.abeclin.org.br/post.php?p=213.

9. Gil AC. Como elaborar um projeto de pesquisa. São Paulo: Atlas; 2010.

10. Fonseca JJS. Metodologia da pesquisa científica. Fortaleza: UEC; 2002.

11. Ruktanonchai NW, Floyd JR, Lai S, et al. Assessing the impact of coordinated COVID-19 exit strategies across Europe. Science. 2020; 369(6510):1465-1470.

12. Mathew D, Giles JR, Baxter AE, et al. Deep immune profiling of COVID-19 patients reveals distinct immunotypes with therapeutic implications. Science. 2020; 369(6508):eabc8511.

13. World Health Organization. Technical specifications for invasive and non-invasive ventilators for $\mathrm{CO}$ VID-19: Interim guidance. Genebra: World Health Organization; 2020. [acesso em 2020 jun 15]. Disponível em: https://apps.who.int/iris/handle/10665/331792.

Recebido em 29/08/2020

Aprovado em 21/07/2021

Conflito de interesses: inexistente

Suporte financeiro: não houve 